\title{
The Role of Chest Computed Tomography in the Work-Up of Patients with Cervical or Endometrial Cancer
}

\author{
Serap Demir ${ }^{1,2}$, Johanna M. A. Pijnenborg, ${ }^{1,3 \#}$, Ruud L. M. Bekkers ${ }^{1}$ \\ ${ }^{1}$ Department of Obstetrics and Gynecology, Radboud University Medical Center, Nijmegen, The Netherlands; ${ }^{2}$ Department of Cardi- \\ ology, Canisius Whilhelmina Hospital, Nijmegen, The Netherlands; ${ }^{3}$ Department of Obstetrics and Gynecology, TweeSteden Hospital, \\ Tilburg, The Netherlands. \\ Email: "H.Pijnenborg@planet.nl
}

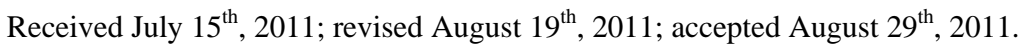

\begin{abstract}
Background and Objectives: To determine whether chest CT-scan in patients with cervical or endometrial cancer is of additional value for planning initial treatment and work-up. Methods: A retrospective analysis was performed of 465 patients diagnosed with cervical or endometrial cancer between January 2003 and December 2007. All patients who underwent a chest CT-scan before treatment were included for analysis. Results: Out of 465 patients 74 patients underwent a pre-treatment chest CT-scan (cervical cancer, $n=58$, and endometrial cancer, $n=26$ ). Abnormalities were detected in $53.4 \%$ (31/58) and $73.1 \%$ (19/26) of patients with cervical and endometrial cancer, respectively. The majority of abnormalities were presumed to be benign, yet work-up was adjusted in 28.6\% (13/58 and 11/26), and therapy was adjusted in $17.6 \%(6 / 58$, and 7/26) of patients with cervical and endometrial cancer. Pulmonary metastasis were observed in 10.3\% (6/58) and 24.1\% (7/26) of patients with cervical cancer, and endometrial cancer, respectively. Most patients with pulmonary metastasis presented with extended disease based on clinical examination. Conclusions: Chest CT scans in patients with cervical and endometrial cancer frequently demonstrate abnormalities that are most likely benign, yet work-up is adjusted in a substantial number of cases. Therefore, chest CT-scan is only recommended for those patients with a clinical suspicion of extended disease.
\end{abstract}

Keywords: Chest CT-Scan, Endometrial Cancer, Cervical Cancer, Work-Up

\section{Introduction}

Endometrial and cervical cancer are the most frequently diagnosed malignancies of the female genital tract. They account for respectively $5.8 \%$, and $1.6 \%$, of all new cancers in women in the United States [1]. Gynaecological tumours are classified according to the FIGO staging system, and for both endometrial and cervical cancer, FIGO stage is the most important prognostic factor [2,3]. Staging of cervical cancer is performed by a clinical appraisal preferably confirmed with a patient under anaesthesia. Determination of local spread and a biopsy of the, lesion are necessary for clinical staging. Additional im-

\footnotetext{
"Synopsis for Table of Contents: A retrospective analysis of the impact of chest CT-scan in the work-up of patients diagnosed with cervical or endometrial cancer was performed. Even in clinical stage I disease abnormalities on chest CT-scan are frequently found but are mostly benign. Therefore, chest CT-scan is only recommended for those patients with extended disease on clinical examination.
}

aging is optional and depends on the extend of the disease. Endometrial cancer on the contrary, is surgically staged by laparotomy, or laparoscopy, and includes a total abdominal hysterectomy, bilateral salpingo-oophorectomy, and palpation/dissection of pelvic and paraaortic lymph nodes [4-6]. For both cervical and endometrial cancer a chest $\mathrm{X}$-ray is recommended as initial tool to exclude pulmonary metastasis [6,7]. However, when compared with computed tomography (CT), chest $\mathrm{X}$-ray is less sensitive for detecting abnormalities [8-10]. Yet, due to its high sensitivity, chest CT will also identify abnormalities of which it is unclear whether these are related to the primary malignant process. CT of the abdomen is frequently performed in the work-up for the detection of lymphadenopathy additional to conventional staging, extended scanning including the chest is then easily performed $[3,11]$. In order to determine the incidence of the abnormalities observed on the pre-treatment 
CT-scan of the chest and the influence of these abnormalities on the primary treatment and further work-up, a retrospective analysis was performed in patients with cervical and endometrial cancer.

\section{Material and Methods}

\subsection{Study Population}

Retrospectively, all patients diagnosed with endometrial or cervical cancer at the Radboud University Nijmegen Medical Center, from January 2003 till December 2007 were selected and subjected to analysis. A total of 465 patients were reviewed; 290 patients with cervical cancer, and 175 patients with endometrial cancer. The medical records were reviewed for information about the age, tumor stage, initial therapy, tumor histology and grade, and tumor characteristics. Only patients who underwent a chest CT-scan before the primary treatment were subjected to further analysis.

\subsection{Chest CT-Scan}

Reports of the performed chest CT-scan were collected and read carefully. In case of inconsistency between the description of the CT-scan report and the final conclusion, revision was performed by an expert radiologist. Findings on chest CT-scan were divided into four categories: no abnormalities (A), benign abnormalities (B), pulmonary metastasis (C), doubtful malignancy (D). Moreover, it was documented whether: treatment was adjusted (I), additional investigations were done (II), follow-up CT-scans were performed (III) or no further actions were taken (IV).

\subsection{Statistical Analysis}

Statistical analysis was performed using the SPSS (version 16). The statistical tests used in evaluating the data were Pearson chi-square, Fishers's exact method, and one-sample T test. Age, tumor stage, and histopathology were compared of those patients with and without a chest CT scan. A $p$-value of $<0.05$ was considered statistically significant.

\section{Results}

\subsection{Study Population and Tumor Characteristics}

A total of 465 patients were diagnosed with endometrial or cervical cancer during the study period. A chest CT scan was performed in $20 \%$ (58/290) of patients with cervical cancer and in 15\% (26/175) of patients with endometrial cancer. Chest CT-scan was mostly performed in conjunction with an abdominal CT and not as a separate investigation.

\subsection{Patients with Cervical Cancer}

In the selected group of patients with a pre-treatment chest CT-scan, median age was not statistically significant when compared to those patients without a chest CT-scan. Yet, there was an increased proportion of patients with stage III/IV disease in the group with a chest CT-scan. In four out of the seven patients with stage IV, extended disease was already suspected at initial gynecological examination. Regarding tumor histology, there was a normal distribution of squamous cell carcinoma (77.6\%) and adenocarcinoma (20.7\%) in the selected population. However, an increased percentage of poorly differentiated tumors was observed in the group of patients with a performed chest CT-scan. Unfortunately, tumor differentiation was unknown in a substantial number of patients (Table 1). Cervical cancer patients in the chest CT-scan group were treated with: surgery (55.2\%), radiation (10.3\%), chemotherapy (6.9\%), chemoradiation (17.2\%), combined surgery and chemotherapy (3.4\%), and combined surgery and radiation (1.7\%).

\subsection{Chest CT-Scan of Patients with Cervical Cancer}

Abnormalities on chest CT-scan were observed in 53.4\% (31/58) (Table 2). The majority of these abnormalities were presumed to be benign lesions. Pulmonary metastases were present in four patients with cervical cancer. In those patients without abnormalities on chest CT-scan, two patients developed pulmonary metastasis during follow-up. Treatment was adjusted in $10.3 \%$ (6/58): four patients with pulmonary metastasis and two patients with benign abnormalities. Additional investigations or follow-up CT-scans were performed in nine patients (15.5\%), all with presumed benign abnormalities. Additional investigations consisted of lymph node biopsy $(n=1)$ and positron emission tomography (PET-scan) $(n=2)$. Revision of the Chest CT was performed in eight patients and changed the final conclusion in two patients with a doubtful malignancy into a presumable benign lesion.

\subsection{Patients with Endometrial Cancer}

The median age in the group of patients with a chest CT-scan was not significantly different when compared to the patients without a chest CT-scan. However, there were significantly more patients with stage IV disease in the group with a chest CT-scan (Table 3). In four out of these five patients, extended disease was already observed at clinical examination. The most frequent tumor histology type was endometrioid adenocarcinoma (50\%). Tumor differentiation was unknown in a large number of patients. Yet, chest CT-scans were significantly more performed in those patients with poorly differentiated 
Table 1. Patient and tumor characteristics of patients with cervical cancer.

\begin{tabular}{ccccc}
\hline Patients with cervical cancer & $\begin{array}{c}\text { Total population } \\
\text { (n = 290) }\end{array}$ & $\begin{array}{c}\text { Patients with Chest CT-scan } \\
\text { (n = 58) }\end{array}$ & $\begin{array}{c}\text { Patients without Chest CT-scan } \\
\text { (n = 232) }\end{array}$ & P value* \\
\hline Age (median) & $52.7(25-90)$ & $51.7(30-82)$ & $52.9(25-90)$ & \\
FIGO stage & & & & \\
I & $183(63.1 \%)$ & $33(56.9 \%)$ & $50(64.7 \%)$ & 0.64 \\
II & $66(22.8 \%)$ & $11(19.0 \%)$ & $13(5.6 \%)$ & 0.37 \\
III & $20(6.9 \%)$ & $7(12.1 \%)$ & $9(3.9 \%)$ & 0.18 \\
IV & $16(5.5 \%)$ & $7(12.1 \%)$ & $5(2.2 \%)$ & 0.07 \\
Unknown & $5(1.7 \%)$ & $0(0 \%)$ & $11(4.7 \%)$ & 0.31 \\
Tumor differentiation & & & $66(28.4 \%)$ & 0.37 \\
Well differentiated & $16(5.5 \%)$ & $5(8.6 \%)$ & $56(24.1 \%)$ & 0.60 \\
Moderately differentiated & $85(29.3 \%)$ & $19(32.8 \%)$ & $99(42.7 \%)$ & 0.09 \\
Poorly differentiated & $78(26.9 \%)$ & $22(37.9 \%)$ & & \\
Unknown & $111(38.3 \%)$ & $12(20.7 \%)$ & & 0.11 \\
\hline
\end{tabular}

${ }^{*} \mathrm{P}(<0.05$ is significant) for the difference between the population with chest CT-scan and total population.

Table 2. Findings on chest CT-scan in patients with cervical cancer and alterations in work-up.

\begin{tabular}{|c|c|c|c|c|}
\hline Patients with cervical cancer & $\begin{array}{c}\text { I } \\
\text { Adjustment of Therapy }\end{array}$ & $\begin{array}{c}\text { II } \\
\text { Additional investigation }\end{array}$ & $\begin{array}{c}\text { III } \\
\text { Follow-up chest CT-scan }\end{array}$ & $\begin{array}{c}\text { IV } \\
\text { No further work-up }\end{array}$ \\
\hline No abnormalities & & & & $27^{*}$ \\
\hline Benign abnormalities & 2 & 2 & 5 & 17 \\
\hline Pulmonary metastasis & $4^{* *}$ & 1 & 1 & \\
\hline Doubtful malignancy & & & & 1 \\
\hline Total & 6/58 (10.3\%) & 3/58 (5.2\%) & 6/58 (10.3\%) & 45/58 (77.6\%) \\
\hline
\end{tabular}

*Two patients presented with pulmonary metastasis after normal pre-treatment chest CT-scan; ${ }^{* * *}$ Two patients both had adjustment of therapy and additional investigation $(\mathrm{n}=1)$, and follow-up chest CT-scan $(\mathrm{n}=1)$.

tumors (Table 3).

The majority of this selected group of endometrial cancer patients was treated with surgery (73.1\%). Only a small number of patients were treated with radiation (7.7\%), chemotherapy (7.7\%), hormone therapy (3.8\%) or a combination of surgery and radiation (3.8\%).

\subsection{Chest CT-Scan of Patients with Endometrial Cancer}

Abnormalities on chest CT-scan were observed in 73.1\% (19/26) (Table 4). Seven out of these 19 patients presented with pulmonary metastasis on chest CT-scan. Treatment was adjusted in 26.9\% (7/26): six patients with pulmonary metastasis and one patient with a doubt- ful malignancy. Additional investigations/follow-up CTscans were performed in eight patients (30.8\%). Three with presumably benign lesions, one patient with a doubtful malignancy, and in four patients with pulmonary metastasis. Revision of chest CT-scans was performed in four patients without any alterations of the conclusion.

\section{Discussion}

In this retrospective analysis, chest CT-scan was performed in the work-up of $20 \%(58 / 290)$ of patients with cervical cancer, and 15\% (26/175) of patients with endometrial cancer, mostly in conjunction with abdominal CT. Abnormalities on chest CT-scan were frequently 
Table 3. Patient and tumor characteristics of patients with endometrial cancer.

\begin{tabular}{|c|c|c|c|c|}
\hline $\begin{array}{c}\text { Patients with endometrial } \\
\text { cancer }\end{array}$ & $\begin{array}{l}\text { Total population } \\
\quad(\mathbf{n}=175)\end{array}$ & $\begin{array}{l}\text { Patients with Chest CT-scan } \\
\qquad(\mathrm{n}=26)\end{array}$ & $\begin{array}{l}\text { Patients without Chest CT-scan } \\
\qquad(\mathrm{n}=149)\end{array}$ & P value ${ }^{*}$ \\
\hline Age (median) & $66.2(36-90)$ & $69.8(55$ - 89) & $65.6(36-90)$ & 0.08 \\
\hline \multicolumn{5}{|l|}{ FIGO stage } \\
\hline I & $80(45.7 \%)$ & $12(46.2 \%)$ & $68(45.6 \%)$ & 0.97 \\
\hline II & $16(9.1 \%)$ & $1(3.8 \%)$ & $15(10.1 \%)$ & 0.37 \\
\hline III & $31(17.7 \%)$ & $5(19.2 \%)$ & $26(17.4 \%)$ & 0.85 \\
\hline IV & 19 (10.9\%) & $5(19.2 \%)$ & $14(9.4 \%)$ & 0.22 \\
\hline Unknown & $29(16.6 \%)$ & $3(11.5 \%)$ & $26(17.4 \%)$ & 0.51 \\
\hline \multicolumn{5}{|l|}{ Tumor differentiation } \\
\hline Well differentiated & $12(6.8 \%)$ & $1(3.8 \%)$ & $11(7.4 \%)$ & 0.56 \\
\hline Moderately differentiated & 20 (11\%) & $5(19.2 \%)$ & 15 (10.1\%) & 0.26 \\
\hline Poorly differentiated & $16(9.1 \%)$ & 7 (26.9\%) & $9(6.0 \%)$ & 0.01 \\
\hline Unknown & $127(38.3 \%)$ & $13(50 \%)$ & $114(76.5 \%)$ & 0.20 \\
\hline
\end{tabular}

${ }^{*} \mathrm{P}(<0.05$ is significant) for the difference between the population with chest CT-scan and the general population.

Table 4. Findings on chest CT-scan in patients with endometrial cancer, and alterations in work-up.

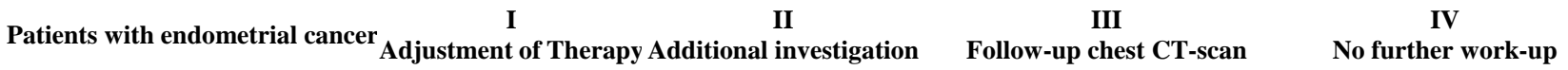

\begin{tabular}{|c|c|c|c|c|}
\hline No abnormalities & 0 & 0 & 0 & 7 \\
\hline Benign abnormalities & 0 & 0 & 3 & 7 \\
\hline Pulmonary metastasis & $6^{*}$ & 1 & 4 & 1 \\
\hline Doubtful malignancy & 1 & 0 & 1 & \\
\hline Total & 7/26 (26.9\%) & $1 / 26(3.8 \%)$ & 6/26 (23.1\%) & $15 / 26(57.7 \%)$ \\
\hline
\end{tabular}

*Four patients had both had adjustment of therapy and follow-up chest CT-scan $(\mathrm{n}=3)$, and had both adjustment of therapy, additional investigation, and follow-up CT scan $(\mathrm{n}=1)$.

seen, but the majority of these findings were presumed to be benign. In the group of patients with a chest CT-scan, therapy was adjusted in $10.3 \%$ of patients with cervical cancer, and in $26.9 \%$ of patients with endometrial cancer, mainly due to the presence of pulmonary metastasis.

To our knowledge, this is the first study to report the influence of chest CT-scan in the work-up on treatment of cervical and endometrial cancer. The most important reason to perform pre-treatment imaging is to evaluate the extension of the disease and involvement of lymph nodes [11-13]. For detection of pulmonary nodules CT is superior to chest X-ray [8-10,14,15]. Although CT is highly specific for a benign lesion, many lesions remain of uncertain significance after CT evaluation, and require histological confirmation of diagnosis $[16,17]$, or follow- up scans.

The most important risk factors for pulmonary metastasis are stage, grade and histology $[18,19]$. Indeed, pulmonary metastasis were more frequently observed in patients with grade III tumors and in patients with advanced disease. In our study the final conclusion in the radiology report was the most important predictor for further investigations, i.e. pulmonary metastasis or doubtful malignancy. For the distinction of benign and malignant lesions; location, diameter, calcification of the lesion, and the number of lesions are important [20]. Irregular and speculated lesions strongly suggest malignancy, whereas lesions with smooth, well-defined margins are more likely to be benign [16,20-23]. The presence and pattern of calcification are strong indicators for 
benignancy $[16,20,21,24]$. Additionally, stability of the lesions for two years, and small size of the lesions $(<2-3$, $5 \mathrm{~mm}$ ) are other factors suggestive of benign disease $[10,16,21,22,24,25]$. After critical reviewing the chest CT scan reports, further investigation was recommended in $50 \%(9 / 18)$ of the patients with cervical cancer. Although there are several recommendations according the follow-up of pulmonary lesions detected on chest CT scan, there is no clear consensus $[19,25]$. Chest CT scan seems to be necessary after a normal chest $\mathrm{x}$-ray, only in patients with a high risk for pulmonary metastasis, and in whom detection of occult metastases would alter the therapy [26].

A new diagnostic tool to detect (pulmonary) metastasis is a PET-CT scan, which is based on increased uptake of fluorodeoxyglucose (FDG) in malignant cells. PET scan is superior in the detection of lymph node metastasis compared to conventional imaging [27-29]. Patz et al. evaluated a variety of focal pulmonary abnormalities by using a FDG PET, and determined a threshold of standardized uptake ratio (SUR) of 2.5 for optimal sensitivity and specificity of benign lesions. With this SUR cut-off value the specificity of detecting a benign lesion was $100 \%$ and the sensitivity was $89 \%$ [30]. When single CT and PET were combined with integrated PET-CT scan, no benefit was observed for the detection of small pulmonary metastasis [31]. Unfortunately, comparison of pre-treatment PET- and chest CT-scan in patients with endometrial and cervical cancer has not been reported yet. The role of FDG PET scan has been studied prospectively in patients with recurrent cervical cancer and did alter treatment in $55 \%-65 \%$ of these patients [32,33]. In two small series of patients with endometrial cancer FDG-PET scan was studied in follow-up and resulted in treatment adjustment in 33\% - 35\% of the patients [34, 35]. According to these studies the contribution of PET scan to treatment planning seems to be superior to CTscan due to its higher sensitivity for the detection of metastasis [32-34,36]. Yet, like CT-scans, the value of a PET scan is limited in the detection of pulmonary micro metastasis [35,37].

The current study is limited by its retrospective character and the possibility of selection bias. In those patients who underwent a chest CT-scan the proportion of patients with FIGO stage III/IV was increased when compared to the patients without a chest CT-scan. Extended disease was already suspected at presentation in most of these patients, and hence could have influenced the request of a chest CT-scan. Moreover, selection bias could be present due to the fact that it was unclear why an additional chest CT-scans was requested. Finally, inconsistencies were observed in the value that was attributed to the observations that were reported on chest CT- scan. As illustrated by our data, both additional investigations and follow-up were performed in presumed benign lesions, which suggest that there was still an uncertainty about a malignancy. Lacking structural assessment and standardization of all morphological abnormalities observed on chest CT-scan, as well as observer variability could contribute to a bias of the study results.

\section{Conclusions}

Concluding, chest CT-scans performed in the work-up of cervical and endometrial cancer frequently demonstrate abnormalities which are mostly benign. Assessment and interpretation of these abnormalities may vary substantially and needs further standardization. Based on our data, chest CT-scan in cervical and endometrial cancer patients is recommended only in those patients with a suspicion of advanced disease based on clinical examination. Future studies are necessary to determine whether new imaging techniques, such as PET-CT are more helpful in diagnosing true metastases, and in preventing unnecessary treatment alterations or additional investigations.

\section{Acknowledgements}

We would like to thank Dr. S. P. Strijk who revised all CT-scans with inconsistency. Dept of Diagnostic Radiology, Radboud University Medical Center.

\section{REFERENCES}

[1] American Cancer Society, "Cancer Facts and Figures 2008,” Atlanta, 2008.

[2] S. Pecorelli, "Revised FIGO Staging for Carcinoma of the Vulva, Cervix, and Endometrium,” International Journal of Gynecology and Obstetrics, Vol. 105, No. 2, 2009, pp. 103-104. doi:10.1016/j.ijgo.2009.02.012

[3] S. E. Waggoner, “Cervical Cancer,” Lancet, Vol. 361, No. 9376, 2003, pp. 2217-2225. doi:10.1016/S0140-6736(03)13778-6

[4] T. Koyama, K. Tamai and K. Togashi, "Staging of Carcinoma of the Uterine Cervix and Endometrium," European Radiology, Vol. 17, No. 8, 2007, pp. 2009-2019. doi:10.1007/s00330-006-0555-0

[5] J. Scheidler and A. F. Heuck, "Imaging of Cancer of the Cervix," Radiologic Clinics of North America, Vol. 40, No. 3, 2002, pp. 577-590. doi:10.1016/S0033-8389(01)00007-0

[6] S. Pecorelli, J. L. Benedet, W. T. Creasman and J. H. Shepherd, "FIGO Staging of Gynecologic Cancer," International Journal of Gynecology and Obstetrics, Vol. 64, 1999, pp. 5-10.

[7] S. C. Ballon, M. L. Berman, R. C. Donaldson, W. A. Growdon and L. D. Lagasse, "Pulmonary Metastases of Endometrial Carcinoma,” Gynecologic Oncology, Vol. 7, No. 1, 1979, pp. 56-65. 


\section{doi:10.1016/0090-8258(79)90081-7}

[8] S. Sone, S. Takashima, F. Li, Z. Yang, T. Honda, Y. Maruyama, et al., "Mass Screening for Lung Cancer with Mobile Spiral Computed Tomography Scanner," Lancet, Vol. 351, No. 9111, 1998, pp. 1242-1245. doi:10.1016/S0140-6736(97)08229-9

[9] C. I. Henschke, D. I. McCauley, D. F. Yankelevitz, D. P. Naidich, et al., "Early Lung Cancer Action Project: Overall Design and Findings From Baseline Screening," Lancet, Vol. 354, No. 9173, 1999, pp. 99-105. doi:10.1016/S0140-6736(99)06093-6

[10] A. E. Chang, E. G. Schaner, D. M. Conkle, et al., "Evaluation of Computed Tomography in the Detection of Pulmonary Metastases. A Prospective Study," Cancer, Vol. 43, No. 3, 1979, pp. 913-916.

[11] O. Akin, S. Mironov, N. Pandit-Taskar and L. E. Hann, "Imaging of Uterine Cancer," Radiologic Clinics of North America, Vol. 45, No. 1, 2007, pp. 167-182. doi:10.1016/j.rcl.2006.10.009

[12] M. Follen, C. F. Levenback, R. B. Iyer, P. W. Grigsby, et al., "Imaging in Cervical Cancer," Cancer, Vol. 98, No. S9, 2003, pp. 2028-2038. doi:10.1002/cncr.11679

[13] R. Iyer, "Imaging of Gynecologic Malignancy," Seminars in Roentgenology, Vol. 39, No. 3, 2004, pp. 428-436. doi:10.1016/j.ro.2004.04.003

[14] J. R. Muhm, L. R. Brown and J. K. Crowe, "Detection of Pulmonary Nodules by Computed Tomography,” American Journal of Roentgenology, Vol. 128, 1977, pp. 267270.

[15] M. Kaneko, K. Eguchi, H. Ohmatsu, et al., "Peripheral Lung Cancer: Screening and Detection with Low-Dose Spinal CT versus Radiography,” Radiology, Vol. 2001, 1996, pp. 798-802.

[16] W. R. Webb, "Radiologic Evaluation of the Solitary Pulmonary Nodules," American Journal of Roentgenology, Vol. 154, 1990, pp. 701-708.

[17] E. A. Zerhouni, F. P. Stitik, S. S. Siegelman, et al., "CT of the Pulmonary Nodule: A Cooperative Study," Radiology, Vol. 160, 1986, pp. 319-327.

[18] A. G. Shumsky, P. M. A. Brasher, G. C. E. Stuart and J. G. Nation, "Risk-Specific Follow-Up for Endometrial Carcinoma Patients,” Gynaecologic Oncology, Vol. 65, 1997, pp. 379-382.

[19] M. Imachi, N. Tsukamoto, T. Matsuyama and H. Nakano, "Pulmonary Metastasis from Carcinoma of the Uterine Cervix,” Gynecologic Oncology, Vol. 33, No. 2, 1989, pp. 189-192. doi:10.1016/0090-8258(89)90549-0

[20] A. W. K. Tang, H. A. Moss and R. J. H. Robertson, "The Solitary Pulmonary Nodule”, European Journal of Radiology, Vol. 45, No. 1, 2003, pp. 69-77. doi:10.1016/S0720-048X(02)00297-8

[21] S. S. Siegelman, F. P. Stitik and W. R. Summer, "Management of the Patient with a Localized Pulmonary Lesion in Pulmonary System,” In: S. S. Siegelman, F. P. Stitik, W. R. Summer and P. O. Alderson, Eds., Practical Approaches to Pulmonary Diagnosis, Grune \& Straton,
New York, 1979, pp. 339-358.

[22] S. S. Siegelman, N. F. Khouri, F. P. Leo, et al., "Solitary Pulmonary Nodules: CT Assessment,” Radiology, Vol. 160, 1986, pp. 307-312.

[23] J. L. Leef III and J. S. Klein, "The Solitary Pulmonary Nodule,” Radiologic Clinics of North America, Vol. 40, No. 1, 2002, pp. 123-143. doi:10.1016/S0033-8389(03)00113-1

[24] D. P. Naidich, E. A. Zerhouni, S. S. Siegelman and R. Moncada, "Computed Tomography of the Thorax," Raven Press, New York, 1984, pp. 171-199.

[25] E. Dinkel, A. Mundinger, D. Schopp, G. Grosser and K. H. Hauenstein, "Diagnostic Imaging in Metastatic Lung Disease,” Lung, Vol. 168, 1990, pp. 1129-1136.

[26] S. D. Davis, "CT Evaluation for Pulmonary Metastases in Patients with Extrathoracic Malignancy,” Radiology, Vol. 180, 1991, pp. 1-12.

[27] Y. Sugawara, A. Eisbruch, S. Kosuma, B. E. Recker, et al., "Evaluation of FDG PET in Patients with Cervical Cancer," The Journal of Nuclear Medicine, Vol. 40, 1999, pp. 1125-1131.

[28] K. Kitajima, K. Murakami, E. Yamasaki, Y. Kaji and K. Sugimura, "Accuracy of Integrated FDG-PET/ContrastEnhanced CT in Detecting Pelvic and Paraaortic Lymph Node Metastasis in Patients with Uterine Cancer,” European Radiology, Vol. 19, No. 6, 2009, pp. 1529-1536. doi:10.1007/s00330-008-1271-8

[29] D. H. Park, K. H. Kim, S. Y. Park, B. H. Lee, C. W. Choi and S. Y. Chin, "Diagnosis of Recurrent Uterine Cervical Cancer: Computed Tomography versus Positron Emission Tomography," Korean Journal of Radiology, Vol. 1, No. 1, 2000, pp. 51-55. doi:10.3348/kjr.2000.1.1.51

[30] E. F. Patz, V. J. Lowe, J. M. Hoffman, et al., "Focal Pulmonary Abnormalities: Evaluation with F-18 Fluorodeoxyglucose PET Scanning,” Radiology, Vol. 188, 1993, pp. 487-490.

[31] W. De Wever, L. Meylaerts, L. De Ceuninck, S. Stroobants and J. A. Verschakelen, "Additional Value of Integrated PET-CT in the Detection and Characterization of Lung Metastases: Correlation with CT Alone and PET Along,” European Radiology, Vol. 17, No. 2, 2007, pp. 467-473. doi:10.1007/s00330-006-0362-7

[32] C. H. Lai, K. G. Huan, L. C. See, T. C. Yen, et al., "Restaging of Recurrent Cervical Carcinoma with Physe [18F]Fluoro-2-Deoxy-D-Glucose Positron Emission Tomography," American Cancer Society, Vol. 100, 2004, pp. 544-552.

[33] T. C. Yen, L. C. See, T. C. Chang, et al., "Defining the Priority of Using 18F-FDG PET for Recurrent Cervical Cancer," Journal of Nuclear Medicine, Vol. 45, 2004, pp. 1632-1639.

[34] T. Saga, T. Higashi, T. Ishimori, M. Mamede, et al., "Clinical Value of FDG-PET in the Follow-Up of PostOperative Patients with Endometrial Cancer, “ Annals of Nuclear Medicine, Vol. 17, No. 3, 2003, pp. 197-203. doi:10.1007/BF02990022 
[35] T. Belhocine, C. De Barsy, R. Hustinx and J. Willems-Foidart, "Usefulness of 18F-FDG PET in the PostTherapy Surveillance of Endometrial Carcinoma,” European Journal of Nuclear Medecine and Molecular Imaging, Vol. 29, No. 9, 2002, pp. 1132-1139.

doi:10.1007/s00259-002-0878-2

[36] A. Husain, T. Akhurst, S. Larson, K. Alektiar, R. R. Barakat and D. S. Chi, "A Prospective Study of the Accuracy of 18Fluorodeoxyglucose Positron Emission Tomo- graphy (18FDG PET) in Identifying Sites of Metastasis Prior to Pelvic Exenteration,” Gynecologic Oncology, Vol. 106, No. 1, 2007, pp. 177-180. doi:10.1016/j.ygyno.2007.03.018

[37] R. Jover, D. Lourido, C. Gonzalez, A. Rojo, L. Gorospe and J. M. Alfonso, "Role of PET/CT in the Evaluation of Cervical Cancer,” Gynecologic Oncology, Vol. 110, No. 3, 2008, pp. S55-S59. doi:10.1016/j.ygyno.2008.05.023 